\title{
Haptic-guided shared control grasping: collision-free manipulation
}

\author{
Soran Parsa*1,2 ${ }^{\text {, Disha Kamale }}{ }^{* 1}$, Sariah Mghames*1,2, Kiyanoush Nazari*1,2, Tommaso Pardi ${ }^{3}$, \\ Aravinda R. Srinivasan ${ }^{4}$, Gerhard Neumann ${ }^{5}$, Marc Hanhaide ${ }^{1}$ and Amir Ghalamzan E. ${ }^{* 1,2}$
}

\begin{abstract}
We propose a haptic-guided shared control system that provides an operator with force cues during reach-tograsp phase of tele-manipulation. The force cues inform the operator of grasping configuration which allows collision-free autonomous post-grasp movements. Previous studies showed haptic guided shared control significantly reduces the complexities of the teleoperation. We propose two architectures of shared control in which the operator is informed about (1) the local gradient of the collision cost, and (2) the grasping configuration suitable for collision-free movements of an aimed pick-andplace task. We demonstrate the efficiency of our proposed shared control systems by a series of experiments with Franka Emika robot. Our experimental results illustrate our shared control systems successfully inform the operator of predicted collisions between the robot and an obstacle in the robot's workspace. We learned that informing the operator of the global information about the grasping configuration associated with minimum collision cost of post-grasp movements results in a reach-to-grasp time much shorter than the case in which the operator is informed about the local-gradient information of the collision cost.
\end{abstract}

\section{INTRODUCTION}

Tele-operating a robotic manipulator (e.g. a telemanipulation setup is shown in Fig. 1), called slave-arm (SA), with a conventional input device, imposes a high cognitive load on expert human operators and, consequently, results in severe fatigue and progressive degeneration in performance [1]. Haptic-guided shared control has been proposed to reduce the cognitive load on the human operator during tele-manipulation [2].

Haptic devices have been used as a master arm (MA) to apply some informative haptic force cues (HFCs) to the operator's hand. For instance, haptic devices have been used to inform an operator of joint limits, singularities and non holonomic cutting constraints or optimal grasping configuration [3]-[6]. Other costs are also used to characterise grasping configuration associated with maximum safety [7] and minimum torque effort [8]. In addition, HFCs are used to inform the operator of the instantaneous collision, singularities, and joint limits in dual-arm manipulation [3] as well as in single-arm manipulation [4] (where a second arm autonomously moves a camera to provide a better view to the operator during telemanipulation). Moreover, instantaneous nonholonomic-like constraints have been used to represent the cutting kinematics [9]. Furthermore, HFCs are utilised to

\footnotetext{
* These authors contributed equally to this work

1 University of Lincoln, Lincoln Centre for Autonomous System; ${ }^{2}$ Intelligent Manipulation Lab; ${ }^{3}$ University of Birmingham; ${ }^{4}$ University of Leeds; ${ }^{5}$ Karlsruhe Institute of Technology. This work was partly funded by NCNR initiative, funded by EPSRC EP/R02572X/1, EPSRC flexible partnership funding on 'Haptic-guided shared control of mobile manipulation task', IUK \#104587 which is part of the RASberry programme;
}

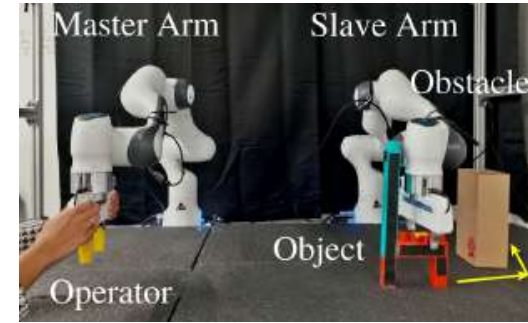

Fig. 1. Tele-operation setup: the human operator moves the master device (a Panda robot at the left of the image) and the slave manipulator (Panda robot at the right of the image) follows the movements of the master one.

notify the operator of singularities and joint limits along a predicted SA trajectory [10]. The above non-conventional use of HFCs approaches significantly improved the teleoperation experience. However, receiving haptic-force cues proportional to the gradient of a pre-specified cost, albeit extremely useful, is sometimes not intuitive and might cause additional cognitive load on the operator [2].

In a conventional tele-manipulation case, an operator steers the slave arm to grasp an object (Fig. 1) and, e.g., performs a Pick-and-Place $(\mathrm{PaP})$ task. Successful completion of the $\mathrm{PaP}$ task is possible by efficient reach-to-grasp execution which allows sufficient post-grasp manipulative movements after forming stable contacts. We assume that the object trajectory which is required to perform the PaP task is given (Fig. 2) by either a reliable planning algorithm [11] or learning from demonstration [12]. State-of-the-art approaches of grasping are used to compute robust grasping configurations given single view point cloud [13] or multiple view point cloud [14] which may be enhanced with an estimated 3-D model of the object [15].

In this paper, we present shared control architectures in which an operator steers a SA to reach an object (reach-tograsp - $g_{0}$ ), form stable contacts between SA fingers and object (grasp synthesis $-g_{1}$ ) and the autonomous system moves the object to the desired position/orientation (postgrasp manipulative movement $-g_{2}$ ). While collision-free grasping has been intensively explored [16], [17], considering post-grasp collision-free movements for choosing a grasp has been mostly ignored [18]. Predicting collision during post-grasp movements is non-intuitive and operators typically perform several grasps, move, re-plan sequences, e.g., this happens many times in robotic surgery [19].

The contribution of this paper is twofold: (1) we present two haptic-guided shared control (HGSC) architectures (Fig. 3) which inform the operator of predicted collision cost during post-grasp movements; (2) we compare two concepts for designing the HGSC: in the first shared control architecture, force cues provide local information about the collision cost whereas in the second shared control architecture force cues 


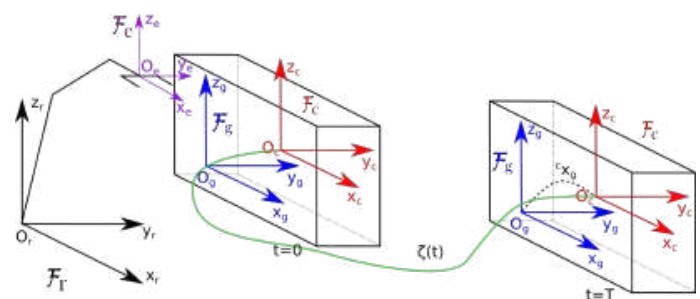

Fig. 2. The initial $(t=0)$ and final state $(t=T)$ of an object trajectory $\zeta(t)$ (shown with a green line): $\mathcal{F}_{e}$ is the robot's end-effector frame, the frame $\mathcal{F}_{c}$ is attached to centre of mass of the object, and a grasping configuration $\mathcal{F}_{g}$ is shown with a frame attached to the object. The constant matrix ${ }^{c} T_{g}$ transforms points in the frame $\mathcal{F}_{g}$ to $\mathcal{F}_{c}$. All frames are expressed using the inertial global frame $\mathcal{F}_{r}$.

provide global information about the collision-free grasping candidate. We performed a series of experiments with two Panda arms used as MA and SA. Our experimental results show both of our proposed shared control architectures successfully help the operator to select a grasping configuration enabling collision-free post-grasp movements. Moreover, our experimental results show that providing the operator with global information about the collision-free grasping candidate results in shorter reach-to-grasp time.

\section{PROBlEM FORMULATION}

We have 2 Panda arms manufactured by Franka Emika as our tele-manipulation setup: Panda-1 is a master arm (MA) and Panda-2 is a slave arm (SA), left and right in Fig. 1. Let $\mathcal{F}_{c}:\left\{O_{c} ; x_{c}, y_{c}, z_{c}\right\}$ denote a local frame attached to the object centre of mass (see Fig. 2), where $\mathcal{F}_{r}:\left\{O_{r} ; x_{r}, y_{r}, z_{r}\right\}$ is the inertial reference frame attached to SA base, and $\mathcal{F}_{e}$ : $\left\{O_{e} ; x_{e}, y_{e}, z_{e}\right\}$ is a local frame attached to the end-effector of the slave arm. Moreover, consider a local frame representing a grasping pose candidate on the object shape denoted by $\mathcal{F}_{g}:\left\{O_{g} ; x_{g}, y_{g}, z_{g}\right\}$. The frame $\mathcal{F}_{c}$ can be expressed in $\mathcal{F}_{r}$ through the transformation matrix ${ }^{1}{ }^{r} x_{c} \in \mathrm{SE}(3)$. The trajectory of the object can thus be expressed by a sequence of planned poses (position and orientation), i.e. $\mathcal{F}_{c}(t) \quad t \in$ $[0, T]$, at every time $0 \leq t \leq T$ and $T$ is the total time to complete a pick-and-place task (see Fig. 2).

The object is not deformable and the SA end-effector forms stable contacts on the object surface, $\mathcal{F}_{e}$ becomes equal to $\mathcal{F}_{g}$ once stable contacts are made. In fact, $\mathcal{F}_{g}$ can be fully expressed at all times during post-grasp movements by a fixed transformation matrix, namely ${ }^{c} x_{g}$, w.r.t. object local frame $\mathcal{F}_{c}$. The slave arm end-effector trajectory for the postgrasp movements can be computed given the planned object trajectory, ${ }^{r} x_{g}(t)=\left\{{ }^{r} x_{c}(t)^{c} x_{g}: 0 \leq t \leq T\right\}$. Finally, the post-grasp joint configuration trajectory corresponding to a given grasp candidate ${ }^{r} x_{e}(t)={ }^{r} x_{g}(t)$, can be computed using the inverse kinematic of SA, i.e., $\hat{\boldsymbol{q}}_{g}(t)=\operatorname{IK}\left({ }^{r} x_{g}(t)\right)$. The problem is to find a good initial grasping configuration expressed by ${ }^{c} x_{g}$ whose corresponding end-effector trajectory allows the IK to produce a collision-free path.

\footnotetext{
${ }^{1}$ In general, ${ }^{b} x_{a} \in \mathrm{SE}(3)$ denotes a homogeneous transformation matrix representing the pose of local frame $a$ in local frame $b$.
}

\section{A. Collision costs for post-grasp movements}

We consider a set of body points attached to manipulator's links ( $\mathbf{b}_{j}=\left\{b_{j, 1} \in \mathbb{N}, b_{j, 2} \in \mathbb{R}\right\}$ where $b_{j, 1}$ denotes the link number to which a body point is attached and $b_{j, 2}$ represents the corresponding distance between the $j_{t h}$ joint and the body point.), e.g. one body point is attached to every joint and one is attached to the middle of every link. Then a group of trajectories expressing the movements of the body points for a known grasping candidate ${ }^{r} x_{g}$, and a given sampling time of a desired post-grasp trajectory are defined. Also, an obstacle is specified by a set of linear constraints and a set of inequalities describing a convex region, as presented in [18]. We use a function that is arbitrarily large in the region inside the obstacle and decreases sharply proportional to $d$ that is the distance between $\mathbf{b}$ and $g_{h}(\mathrm{x})=0$, as per equation 1 .

$$
d_{i}\left(\mathbf{b}_{j}, \mathrm{x}\right)=\sum_{h=1}^{n_{h}}\left\{\hat{d}\left(g_{h}^{i}(\mathrm{x})=0, \mathbf{b}_{j}\right)+\left|\hat{d}\left(g_{h}^{i}(\mathrm{x})=0, \mathbf{b}_{j}\right)\right|\right\}
$$

where $g_{h}(\mathrm{x})$ is a set of linear constraints which specify the obstacle, $X$ is a desired post grasp trajectory for body points, $n_{h}$ is the number of linear constraints and $\hat{d}$ is the distance between $\mathbf{b}$ and the plane $g_{h}(\mathbf{x})=0$. If $\mathbf{b}_{j}$ is on or inside the convex region of the $i_{t h}$ obstacle, then $d_{i}=0$. Hence, we write the obstacle avoidance cost as a function of ${ }^{c} x_{g}$, as per eq. (2).

$$
h\left(\left.{ }^{c} x_{g}\right|^{r} x_{c}, g_{h}\right)=\left|1 /\left(d\left(\left.{ }^{c} x_{g}\right|^{r} x_{c}, g_{h}\right)+\delta\right)\right|_{1}
$$

where $\delta^{-1}$ is a fixed value for setting the maximum available cost. The calculations of total costs, $\mathcal{H}$, for all body points, obstacles and sample points during post-grasp movements are presented in detail in [18].

$\mathcal{H}$ is the integral of $h$ over the post-grasping movements which yields maximum costs on and inside the convex region representing an obstacle. A post-grasp manipulator trajectory that yields the minimum value of $\mathcal{H}$ ideally allows collisionfree movement for the robot. By definition, the value of $\mathcal{H}$ is a function of object trajectory, grasping configuration, and obstacle position and orientation, and shape. Ideally, the operator is expected to steer SA towards a grasping configuration with a low value of collision cost, i.e. ${ }^{c} x_{g}^{*}=$ $\underset{c}{\operatorname{argmin}} \mathcal{H}$. We use the collision cost in the next sections to ${ }^{c} x_{g}$ form the haptic force cues.

\section{B. Haptic-guided 1 (HG-1)}

We consider a stacked vector of the slave arm linear $(\dot{\boldsymbol{p}} \in$ $\left.\mathbb{R}^{3}\right)$ and angular $\left(\boldsymbol{\omega} \in \mathbb{R}^{3}\right)$ velocities during reach-to-grasp ${ }^{2}$ by $\dot{x}_{e}=\left[\dot{\boldsymbol{p}}^{T}, \boldsymbol{\omega}^{T}\right]^{T}$. Combining this with the SA differential forward kinematics, yields

$$
\dot{\boldsymbol{q}}_{s}=\boldsymbol{J}_{s}^{\dagger}\left(\boldsymbol{q}_{s}\right)^{r} \overline{\boldsymbol{R}}_{c}^{c} \dot{x}_{g},
$$

where $\boldsymbol{J}_{s}^{\dagger}$ denotes the usual $\boldsymbol{J}_{s}$ Moore-Penrose pseudoinverse and $\overline{\boldsymbol{R}}_{c}$ is the transformation matrix. Equation (3) will be exploited in the followings to generate haptic-guided force

\footnotetext{
${ }^{2} \dot{x}_{e}={ }^{r} \dot{x}_{g}$ once the end-effector is in contact with object and during
} post-grasp movements. 
cues. Using Leibniz's formulas and the chain rule, we can write

$$
\frac{\partial h}{\partial^{c} x_{g}}=\frac{\partial h}{\partial \boldsymbol{q}_{s}} \boldsymbol{J}_{s}^{\dagger}\left(\boldsymbol{q}_{s}\right)^{r} \overline{\boldsymbol{R}}_{c} .
$$

The partial derivatives of (2) w.r.t. $\boldsymbol{q}_{s}$ can be easily computed and then plugged in (4). (For more details see [2], [5])

We consider a classical bilateral force-guided system to generate some force cues at MA which inform the operator of the cost gradient. The operator interacts with MA by applying some forces to move it. MA is coupled (via velocity-velocity mirroring) with a SA. The master device is modelled as a generic (gravity pre-compensated) mechanical system

$$
\boldsymbol{M}\left({ }^{m} \mathbf{x}_{M}\right)^{m} \ddot{\mathbf{x}}_{M}+\boldsymbol{C}\left({ }^{m} \mathbf{x}_{M},{ }^{m} \dot{\mathbf{x}}_{M}\right)^{m} \dot{\mathbf{x}}_{M}=\overline{\boldsymbol{\tau}}+\boldsymbol{\tau}_{h 1},
$$

where $\boldsymbol{M}\left({ }^{m} \mathbf{x}_{M}\right) \in \mathbb{R}^{6 \times 6}$ is the positive-definite and symmetric inertia matrix, $\boldsymbol{C}\left({ }^{m} \mathbf{x}_{M},{ }^{m} \dot{\mathbf{x}}_{M}\right) \in \mathbb{R}^{6 \times 6}$ consists of Coriolis/centrifugal terms, and $\bar{\tau}, \boldsymbol{\tau}_{h 1} \in \mathbb{R}^{6}$ are the control and human forces, respectively, applied at MA endeffector and ${ }^{m} \mathbf{x}_{M} \in \mathbb{R}^{6}$ represent the Cartesian position and orientation of the master device in MA base frame $\mathcal{F}_{m}$. MA and SA, in our example, have the same kinematics and we use joint position of master $\boldsymbol{q}_{m}$ to slave $\boldsymbol{q}_{s}$ mapping. As such, we use $\boldsymbol{q}$ to represent joint space configuration of MA and SA.

We want the haptic-guided forces inform the user of gradient decent direction of the collision cost. We design force cues $\mathbf{f} \in \mathbb{R}^{6}$ aligned with the negative gradient i.e.,

$$
\boldsymbol{\tau}_{h 1}=-\mathbf{B}^{m} \dot{\mathbf{x}}_{M}-\mathbf{K}_{m} \mathbf{Q}{\frac{\partial \mathcal{H}}{\partial^{c} x_{g}}}^{T}
$$

where $\mathbf{K}_{m}$ is a scaling factor and $\mathbf{Q}$ maps the quaternion rate resulting from the gradient, into a corresponding angular velocity and rotates the result in the master base frame. We add a damping term to the force cues with a positive definite damping matrix $\mathbf{B} \in \mathbb{R}^{6 \times 6}$ to make the force feedback signal feel more stable (see [2] for more details). Fig. 3a shows the block diagram for $H G-1$ strategy. Obstacle poses and grasp candidate pool are received form a Realsense camera and collision costs and cost gradient are calculated and are sent to the MA controller. A PD controller generates haptic-guided forces based on the cost gradient. It is noticeable that all computations in both MA and SA are carried out online.

\section{Haptic-guided 2 (HG-2)}

In this method, instead of generating the haptic force cues proportional to the cost gradient, we form a global shared control strategy to define the force cues. First, we find several grasping configurations $\left(g c=\left\{{ }^{r} x_{g}^{1},{ }^{r} x_{g}^{2}, \ldots,{ }^{r} x_{g}^{n}\right\}\right.$ where $n$ is the number of all grasp candidates) suitable for the pickand-place task using a Realsense camera (which is an RGBD sensor) calibrated w.r.t robot base frame. We calculate off-line the collision cost for each grasp candidate using eq. (2). As such, the grasp candidates resulting in no collision and collision during post-grasp manipulative movements are identified. We introduce (1) attractive force-field and (2)

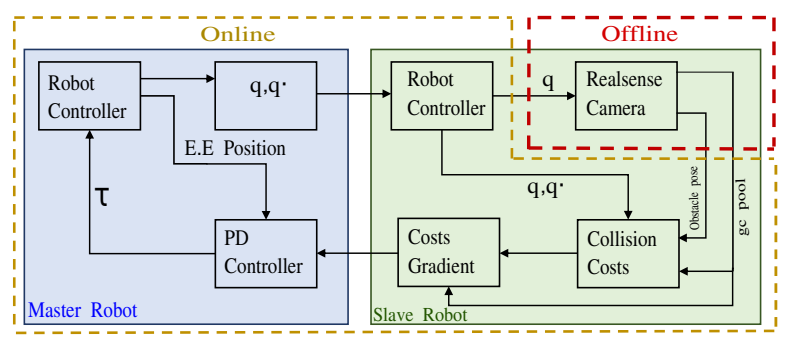

(a)

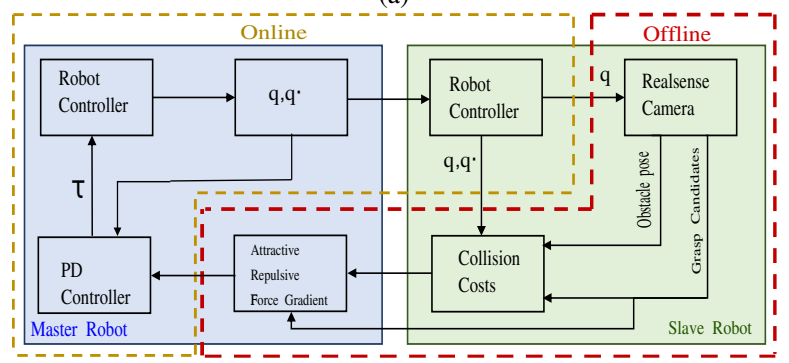

(b)

Fig. 3. 3a shows Schematic block diagrams of $H G-1$ strategy where all computations carried out online, 3b, Schematic block diagrams of $H G-2$ strategy where most of the time consuming computations are carried out offline.

repulsive force-field to inform the operator of the suitable cosponsoring configuration.

In this approach, grasp candidates are categorised into two categories based on their collision costs; a) desired grasp candidate, b) undesired grasp candidate. Median of the collision costs are calculated and grasp candidates with collision costs lower than median considered as the desired grasp candidate and other grasp candidates considered as undesired. We introduce a novel method in which attractive and repulsive haptic forces are defined in joint space. The haptic force $F\left(\hat{M}, \hat{C}, \hat{G}, d_{g c}\right)$ is a function of estimated inertia matrix, $\hat{M}$, Coriolis/centrifugal terms, $\hat{C}$, gravitational forces, $\hat{G}$ and also $d_{g c}$ which is the end effector euclidean distance to the ${ }^{r} x_{g}^{n}$.

The haptic-guided force controller is designed as per eq. (7).

where

$$
\boldsymbol{M}(\boldsymbol{q}) \dot{\boldsymbol{q}}+\boldsymbol{C}(\boldsymbol{q}, \dot{\boldsymbol{q}})+G(\boldsymbol{q})=\hat{\boldsymbol{\tau}}+\boldsymbol{\tau}_{h 2}
$$

$$
\hat{\boldsymbol{\tau}}=\hat{\boldsymbol{M}}(\boldsymbol{q})+\hat{\boldsymbol{C}}(\boldsymbol{q}, \dot{\boldsymbol{q}})+\hat{G}(q)
$$

is the estimated inertia matrix, Coriolis/centrifugal terms and gravitational forces and

$$
\boldsymbol{\tau}_{h 2}=\alpha\left(-k_{v}\left(\dot{\boldsymbol{q}}-\dot{\boldsymbol{q}}_{d}\right)-k_{p}\left(\boldsymbol{q}-\boldsymbol{q}_{d}\right)\right)
$$

Where $k_{v}$ and $k_{p}$ are the PD controller's gains and $\boldsymbol{q}_{d}$ is the joint configuration corresponding to the closest grasping configuration to the end-effector which yields collision-free post-grasp movements. The controller is designed to inform the operator of the grasp candidate nearest to the end effector in the desired category by generating an attractive force field.

Moreover, the controller generates repulsive forces if the operator steers the SA close to a grasping configuration possibly yielding collision during post-grasp movements. In order to generate local repulsive and attractive forces, and help the operator to distinguish between attractive and repulsive 


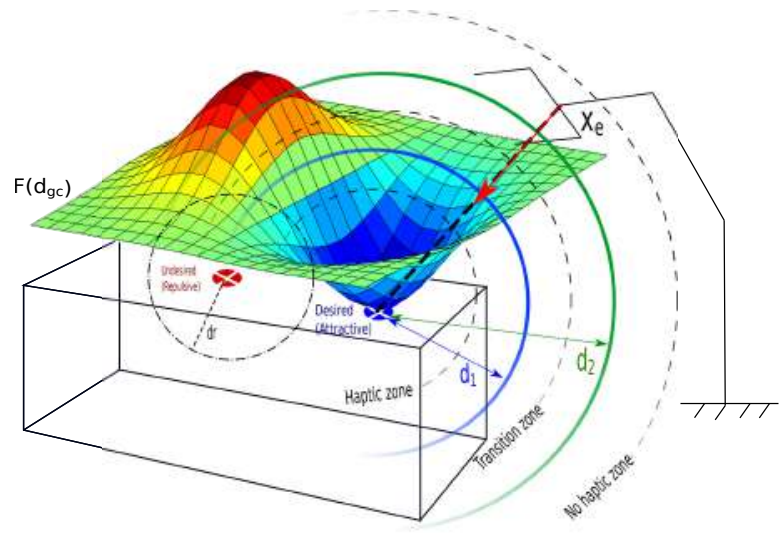

(a)

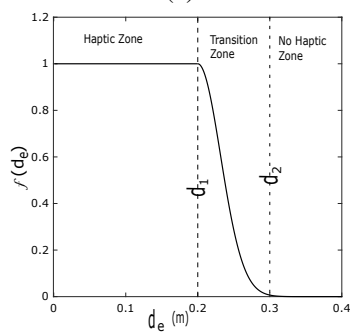

(b)

Fig. 4. 4a Schematic diagram of attractive and repulsive haptic forces. The gradient of the forces around desired (blue point) and undesired (red point) is in a way that attracts end effector toward the desired pose and repels it from the undesired one. Also, operation space around each point divided into three zones as it can be seen. $4 \mathrm{~b}$ shows the transition parameter in different zones which multiples by the haptic forces to generate a smooth and stable transition from no haptic guidance to maximum haptic guidance.

forces, haptic force $\tau_{h 2}$ defined in eq. (7), is multiplied by an exponential function, shown in eq.(10).

$$
\alpha(x)=\frac{1}{d_{r} \sqrt{2 \pi}} e^{-\frac{1}{2}\left(\frac{d_{u}}{d_{r}}\right)^{2}}
$$

Where $d_{r}$ is the distance that the repulsive force is realised by the operator and $d_{u}$ is the euclidean distance between end effector and nearest undesired grasp candidate to end effector.

It is noticeable that in both cases the operator can reach any grasp poses, desired or undesired where attractive and repulsive forces are just informative. The block diagrams in Fig. 3b shows the structure of this strategy where most computational calculations are performed offline. Our hypothesise is that reducing calculation times and therefore increasing control frequency for haptic force generation, lead to more informative haptic forces and reducing cognitive load on the operator. Furthermore, we assume that informing the operator of the suitability of the grasp candidate instead of local information about the local gradient, makes it easier for the operator to select the desired grasp pose in a shorter time.

Figure $4 \mathrm{a}$ shows a schematic diagram of attractive and repulsive haptic forces. For the desired grasp candidate, the blue point is a reference for attractive forces which is proportional to (1) the distance to the joint space and (2) square of Euclidean distance between the end-effector and grasping candidate position. In contrast, for undesired grasp

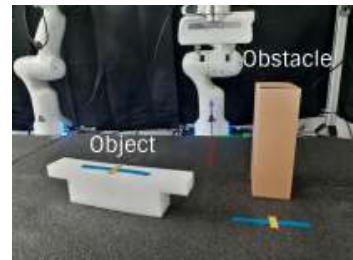

(a)

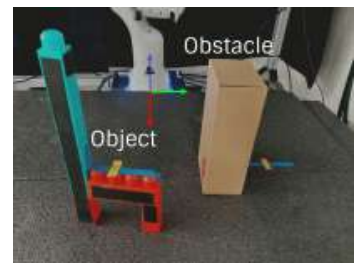

(b)
Fig. 5. Top views of SA workspace for Task I 5a and $25 \mathrm{~b}$. The operator is demanded to move the object 1 in $5 \mathrm{a}$ and object 2 in $5 \mathrm{~b}$ to the final position marked with tape on the table.

candidate, the red point, force's gradient act as a repulsive point which repel the end effector.

To further reduction of cognitive load on the operator, we divided the operation space into three zones. For $d_{e}$, the euclidean distance between the end-effector and its initial nearest desired grasp candidate, if $d_{e}>d_{2}$ no HG force zone, if $d_{1}<d_{e}<d_{2}$ transition zone and if $d_{e}<d_{1}$ haptic-guided zone are defined (as shown in Fig. 4a) where $d_{1}$ and $d_{2}$ are specified based on the object shape and grasp candidates. In no HG force zone, no haptic forces are generated and just robot arm's dynamic and gravitational forces are compensated and operator feels no forces on the end effector. In transition zone, HG forces are gradually increased from zero to its maximum value. Therefore, the calculated $\mathrm{HG}$ force from eq. (7) is multiplied by eq. (11) to generate $\mathrm{HG}$ forces in the transition zone.

$$
f\left(d_{e}\right)= \begin{cases}e^{-\sigma\left(d_{e}-d_{1}\right)^{2}}, & \text { if } d_{e}>d_{1} \\ 1, & d_{e}<d_{1} .\end{cases}
$$

Where $d_{1}=0.2[\mathrm{~m}]$ and $d_{2}=0.3[\mathrm{~m}]$ are radios of haptic and transition zones and $\sigma$ is a constant and in this work $\sigma=500$. In haptic-guided zone, the controller is fully operated and generates HG forces. Fig. 4b shows the graph of eq. (11) which multiplies by the generated haptic forces in different zones.

\section{RESULTS AND DISCUSSION}

\section{A. Experiment set up}

We use 2 Panda arms manufactured by Franka Emika to show the effectiveness of our approach. Panda is a 7 DoF manipulator provided with a control interface, called FCI, and an interface with Robot Operating System (ROS).

Fig. 1 shows our experimental setup consisting of the master arm (MA)-left arm- and slave arm (SA)-right arm. Our teleoperation setup includes a compliant control and a low latency communication between MA and SA using the FCI. These allow a human operator to move the MA easily (creating a feeling similar to a standard 6-DoF haptic device).

The operator stands in front of the MA (see Fig. 1) and looks at their right to the SA. The operator easily steers the MA while the SA is following the movements of the MA. The operator is asked to steer the SA and grasp the object shown in Fig. 5. The post-grasp movements are performed autonomously. We experimented two $\mathrm{PaP}$ tasks: the operator steers SA to pick the object up after which (Task I)- the 


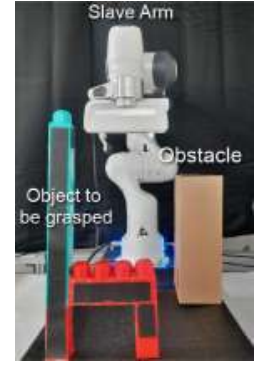

(a) Initial pose

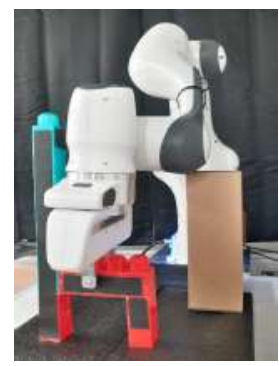

(b) Collision

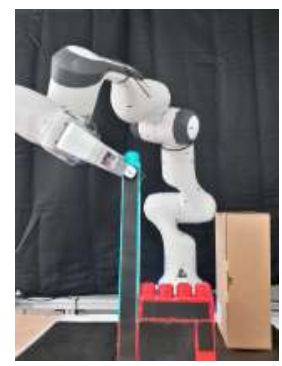

(c) Good grasp
Fig. 6. Snapshots of the SA workspace during experimenting Task II: $6 \mathrm{a}$ shows the SA, object and obstacle at the beginning of Task II experiments; $6 \mathrm{~b}$ shows SA link next to its wrist collides with the obstacle during performing Task II by a chosen grasp on the horizontal bar of the object; $6 \mathrm{c}$ shows collision free movements of the SA by a good choice of grasp.

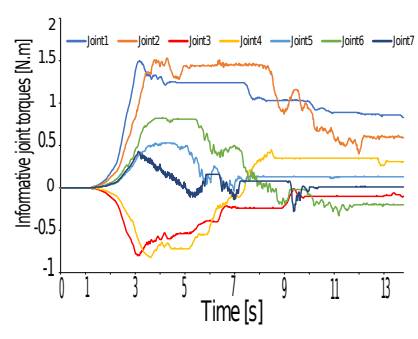

(a)

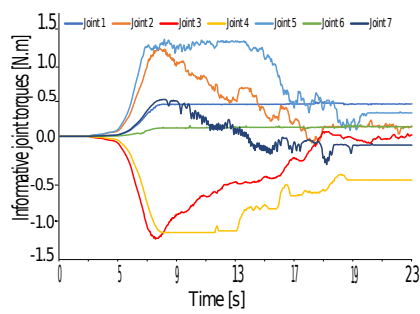

(c)

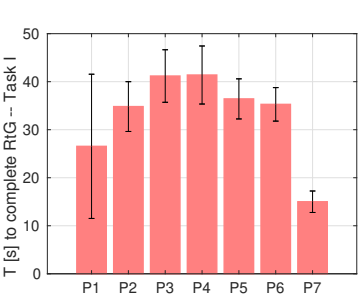

(b)

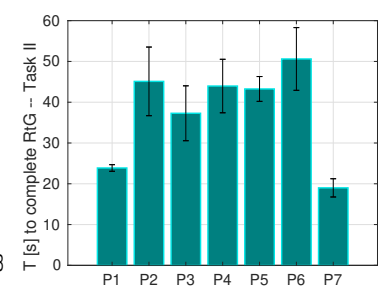

(d)
Fig. 7. 7a and 7c show informative joint torques for the task I and task II respectively using Haptic-guided strategy 2. $7 \mathrm{~b}$ and $7 \mathrm{~d}$ show the mean time-to-completion for the task I and task II respectively where P1-P6 are the policy used with strategy 1 and P7 with strategy 2. (error bars show the standard deviation of the data).

SA autonomously moves the object (Fig. 5a) along $y$ axis of robot base frame (shown in Fig. 4) and places it close to the obstacle; (Task II)-the SA first moves $h$ shaped object in Fig. 5b along $y$ close to the obstacle and then moves it parallel to the obstacle edge, then along $-x$ and finally places it behind the obstacle. We only provide the operator with the target position of the object and they decide which grasping configuration results in collision free post-grasp movements. We designed Task II to be more challenging as the vertical bar of the object, yielding non-intuitive grasping, is the only area on which grasping yield no collision.

We performed both tasks with and without haptic-guided forces (HGF). Although HGF helped the operator during the experiments to understand which grasping configuration yields a lower value of collision cost, our experiments revealed the force cues also distract the operator from the main task, i.e. reach-to-grasp $\left(g_{0}\right)$. The distraction is caused due to the fact that an optimum value to the collision cost $\left(\mathcal{H}_{1}\right)$ does not fully describe $g_{0}$. To fully describe $g_{0}$, we also need to define the actions resulting in stable contacts between SA fingers and object surface. In our control scheme, the human operator is responsible for making stable contacts. As such, optimal actions for minimising collision cost and reaching to grasp may not agree. For instance, the operator wants to move MA along $[1,0,0]$ and the force cues direct the operator's hand along $[0.5,-0.5,0]$.

\section{B. Haptic-guided 1 strategy}

To mitigate this distraction, we propose a set of approaches. we define the projection of the HGFs normal $\left(\boldsymbol{\tau}_{n}\right)$ and tangent $\left(\boldsymbol{\tau}_{t}\right)$ to $B \in \mathbb{R}^{6}$.

$$
\begin{array}{r}
\boldsymbol{\tau}_{t}=<\boldsymbol{\tau}, B> \\
\boldsymbol{\tau}_{n}=\boldsymbol{\tau}-\boldsymbol{\tau}_{t}, \\
\boldsymbol{\tau}_{u_{c}}=\boldsymbol{\tau}_{n}, \\
\boldsymbol{\tau}_{u_{n}}=\left\{\boldsymbol{\tau}_{n}-\boldsymbol{\tau}_{t} \mid\left(\boldsymbol{\tau}_{t} \cdot B\right)<0\right\}
\end{array}
$$

where $\langle A, B\rangle=(A \cdot \bar{B}) B$ and $\bar{B}=\frac{B}{|B|}$ and $\cdot$ denotes the inner product of two vectors. The new proposed HGFs $\tau_{u_{c}}$ and $\tau_{u_{n}}$ cancel and negate the components of the HGFs which are along with $B$. We have two hypotheses to distinguish the distractive elements of the HGFs:

- We denote the vector connecting the end-effector of SA to center of the mass (CoM) by $X_{C G} . B=X_{C G}$ in eq. (12) define the distractive elements of the HGFs. Negating $\left(\boldsymbol{\tau}_{u_{c}, 1}\right)$ and canceling $\left(\boldsymbol{\tau}_{u_{n}, 1}\right)$ the projection of HGFs on $X_{C G}$ in eq. (12) remove the repulsive element of the force.

- The elements of the HGFs along negative movements of the SA distract the operator from reach-to-grasp movements. Thus, $B={ }^{m} \dot{\mathbf{x}}_{M}$ removes distracting terms by canceling $\left(\boldsymbol{\tau}_{u_{c}, 2}\right)$ and negating $\left(\boldsymbol{\tau}_{u_{n}, 2}\right)$ those elements in eq. (12).

Five times experiments were repeated with the first strategy including $5 \mathrm{HGF}$ policies for Task I and II: (P1) with no haptic force cues $(\tau=0)$; (P2) conventional haptic-guided forces, as per (12); (P3) making the tangent element along the $X_{C G}$ attractive $\left(\tau_{u_{n}, 1}\right)$; (P4) cancel the tangent element of the force cues along the $X_{C G}\left(\tau_{u_{c}, 1}\right)$; (P5) making the tangent element along the $\dot{X}_{e e}$ attractive, $\left(\tau_{u_{n}, 2}\right)$; (P6) negate the tangent element of the force cues along $\dot{X}_{e e}\left(\boldsymbol{\tau}_{u_{c}, 2}\right)$.

Task I and II are intentionally selected to represent two extreme cases: (Task I) the intuitive grasping is collision-free (but close to collision) during post-grasp movements; (Task II) the intuitive grasping yields collision. Figs. 7b, 7d show timeto-completion for Task I and Task II respectively. Time to completion of Task I (Fig. 7b) for P1 is a bit less than P2-P6. This confirms the operator experiences almost the same level of complexity of teleoperation with P1 and P2-P6, despite they have no clue of the suitability of the grasping point. The experiments with the task I indicate that the conventional haptic-guided forces (P2) and canceling the projection of HGFs along with the velocities of the MA end-effector (P6) yields the best performance and lowest time-to-completion among the policies of the HG-1 strategy.

Time to completion of Task II for policies P1-P6 are shown in Fig. 7d. Although the time to completion of policy with 
no HGF is almost 50 percent less than the other policies, we observe that the operator reaches the horizontal section of the object which does not provide a collision free grasp point. The results show that making the tangent element along the $X_{C G}$ attractive (P3) results in the best performance and lowest time among the policies of the HG-1 strategy.

\section{Haptic-guided 2 strategy}

The downside of the previous method is that most of the computations are calculated in real-time and it takes 0.2 second on average. It means that produced control torques are updated with $5 \mathrm{~Hz}$ rate which makes real-time controlling problematic. To overcome this problem, we also tested Task I and Task II experiments with HG-2 strategy. In this method, most of the computation are carried out offline to increase the control rate as shown in Fig. $3 b$.

We experimented task I and II, each 5 times, with strategy II, where the corresponding average time-to-completions are shown with P7 in Figs. 7b (task I) and Fig. 7d (task II). The results show 28\% (task I) and 24\% (task II) reduction in reach-to-grasp time compared to no haptic force cases because the generated HGFs guide the operator directly to the nearest good grasping pose. Moreover, the generated control torques for helping the operator are sensed more intuitively. In cases that the nearest grasping configuration is undesired, repulsive forces immediately informs the operator and control torques help the operator to move away from undesired grasping pose. Fig. 7a and 7c show informative torques (without dynamic and gravitational torque compensation). They show joint torques from $\mathrm{t}=0$ where operator starts reach to grasp operation. The end effector is initially out of grasping zone, hence, the informative torques are zero. A smooth transition from zero to maximum informative torques are designed when the endeffector enters the grasping zone. By approaching the end effector position to desired grasping pose, the informative torques converging to zero informing the operator of reaching the desired grasp pose and allowing to perform grasping operation.

Our results in Fig. 7 confirm that our haptic-guided controller successfully informs the operator of a predicted collision cost. Our novel control policy of attractive and repulsive haptic-guided forces yields a significant reduction of time-to-completion and reduced cognitive load on a human operator. This paper is accompanied by a video which could be accessed through the below link. ${ }^{3}$

\section{CONCLUSION}

We presented haptic-guided shared control strategies for tele-operating a manipulator. Our proposed shared control provides the operator with the possible collisions during post-grasp movements and helps the operator to select the grasping configuration suitable for the aimed collision-free movements of a pick-and-place task. We proposed two architectures which (1) computes the force cues proportional to the gradient of collision cost calculated in real-time and

${ }^{3}$ Link to the video of the experiments.
(2) computes the force cues as a PD controller proportional to the Euclidean distance between the end-effector and desired grasping configuration allowing collision-free postgrasp movements. We presented a series of experiments with the Panda teleoperation system. Our experimental results show our shared control systems successfully inform the operator of predicted collisions between the robot and an obstacle in the robot's workspace where the second shared control strategy results in much smoother reach-to-grasp experiments in a much shorter time.

\section{REFERENCES}

[1] M. Talha and et al., "Towards robotic decommissioning of legacy nuclear plant: Results of human-factors experiments with tele-robotic manipulation, and a discussion of challenges and approaches for decommissioning," in IEEE International Conference on Safety, Security, and Rescue Robotics, 2016, pp. 166-173.

[2] A. Ghalamzan and et al., "Human-in-the-loop optimisation: Mixed initiative grasping for optimally facilitating post-grasp manipulative actions," in IEEE/RSJ International Conference on Intelligent Robots and Systems (IROS), 2017, pp. 3386-3393.

[3] M. Selvaggio and et al., "Haptic-based shared-control methods for a dual-arm system," IEEE Robotics and Automation Letters, vol. 3, no. 4, pp. 4249-4256, 2018.

[4] F. Abi-Farraj and et al., "A visual-based shared control architecture for remote telemanipulation," in IEEE/RSJ International Conference on Intelligent Robots and Systems (IROS), 2016, pp. 4266-4273.

[5] M. Selvaggio and et al., "Haptic-guided shared control for needle grasping optimization in minimally invasive robotic surgery," in IEEE/RSJ International Conference Intelligent Robotic System, 2019.

[6] M. Adjigble and et al., "An assisted telemanipulation approach: Combining autonomous grasp planning with haptic cues," in IEEE/RSJ International Conference on Intelligent Robots and Systems, 2019.

[7] N. Mavrakis and et al., "Safe robotic grasping: Minimum impactforce grasp selection," in 2017 IEEE/RSJ International Conference on Intelligent Robots and Systems (IROS), 2017, pp. 4034-4041.

[8] A. Ghalamzan and et al., "Grasp that optimises objectives along postgrasp trajectories," in IEEE International Conference on Robotics and Mechatronics (ICRoM), 2017, pp. 51-56.

[9] R. Rahal and et al., "Haptic shared-control methods for robotic cutting under nonholonomic constraints," in IEEE/RSJ International Conference on Intelligent Robots and Systems (IROS), 2019.

[10] N. Pedemonte and et al., "Visual-based shared control for remote telemanipulation with integral haptic feedback," in IEEE International Conference on Robotics and Automation (ICRA), 2017, pp. 5342-5349.

[11] R. Jackson and et al., "Needle path planning for autonomous robotic surgical suturing," in IEEE Robotic Automation Letter, 2013, pp. 16691675.

[12] S. Schaal and et al., "Learning movement primitives," in International Symposium on Robotics Research. Springer, 2005, pp. 561-572.

[13] M. Kopicki and et al., "Learning better generative models for dexterous, single-view grasping of novel objects," The International Journal of Robotics Research, vol. 38, no. 10-11, pp. 1246-1267, 2019.

[14] T. Pas and et al., "Grasp pose detection in point clouds," The International Journal of Robotics Research, vol. 36, no. 13-14, pp. 1455-1473, 2017.

[15] L. Baronti and et al., "Primitive shape fitting in point clouds using the bees algorithm," Applied Sciences, vol. 9, no. 23, p. 5198, 2019.

[16] N. Vahrenkamp and et al., "Simultaneous grasp and motion planning: Humanoid robot armar-iii," IEEE Robotics \& Automation Magazine, vol. 19 , no. 2, pp. 43-57, 2012.

[17] P. Chotiprayanakul and et al., "Collision-free trajectory planning for manipulators using virtual force based approach," in Proceedings of the International Conference on Engineering, Applied Sciences, and Technology, 2007, pp. 21-23.

[18] T. Pardi and et al., "Choosing grasps to enable collision-free post-grasp manipulations," in 2018 IEEE-RAS 18th International Conference on Humanoid Robots (Humanoids). IEEE, 2018, pp. 299-305.

[19] G. Fontanelli and et al., "A new laparoscopic tool with in-hand rolling capabilities for needle reorientation," IEEE Robotic Automation Letter, vol. 3, no. 3, pp. 2354-2361, 2018. 